\title{
Diagnostic reliability of magnetic resonance imaging for central nervous system syndromes in systemic lupus erythematosus: a prospective cohort study
}

\author{
Yasuhiro Katsumata ${ }^{1 *}$, Masayoshi Harigai ${ }^{1,2}$, Yasushi Kawaguchi', Chikako Fukasawa', Makoto Soejima', \\ Tokiko Kanno', Katsuji Nishimura', Takayuki Yamada4, Hisashi Yamanaka', Masako Hara'
}

\begin{abstract}
Background: Previous studies of magnetic resonance imaging (MRI) as a diagnostic tool for central nervous system (CNS) syndromes in systemic lupus erythematosus (SLE) contained several limitations such as study design, number of enrolled patients, and definition of CNS syndromes. We overcame these problems and statistically evaluated the diagnostic values of abnormal MRI signals and their chronological changes in CNS syndromes of SLE.

Methods: We prospectively studied 191 patients with SLE, comparing those with $(n=57)$ and without $(n=134)$ CNS syndrome. CNS syndromes were characterized using the American College of Rheumatology case definitions.

Results: Any abnormal MRI signals were more frequently observed in subjects in the CNS group $(n=25)$ than in the non-CNS group $(n=32$ ) [relative risk (RR), 1.7; 95\% confidence interval $(\mathrm{Cl}), 1.1-2.7 ; p=0.016$ ] and the positive and negative predictive values for the diagnosis of CNS syndrome were $42 \%$ and $76 \%$, respectively. Large abnormal MRI signals $(\varnothing \geq 10 \mathrm{~mm}$ ) were seen only in the CNS group $(n=7 ; \mathrm{RR}, 3.7 ; \mathrm{Cl}, 2.9-4.7 ; p=0.0002)$, whereas small abnormal MRI signals $(\varnothing<10 \mathrm{~mm}$ ) were seen in both groups with no statistical difference. Large signals always paralleled clinical outcome $(p=0.029)$, whereas small signals did not $(p=1.000)$.

Conclusions: Abnormal MRI signals, which showed statistical associations with CNS syndrome, had insufficient diagnostic values. A large MRI signal was, however, useful as a diagnostic and surrogate marker for CNS syndrome of SLE, although it was less common.
\end{abstract}

\section{Background}

Central nervous system (CNS) lupus is a serious and potentially life-threatening manifestation of systemic lupus erythematosus (SLE), occurring in $37-95 \%$ of cases, and is associated with an increased risk of death [1]. Despite its frequency and severity, the lack of a diagnostic gold standard makes it challenging to differentiate primary CNS lupus from secondary neuropsychiatric (NP) manifestations unrelated to SLE at their onsets [1-3]. The American College of Rheumatology (ACR) has developed a standardized nomenclature system that provides case definitions for 19 NP syndromes

\footnotetext{
* Correspondence: katsumata@ior.twmu.ac.jp

${ }^{1}$ Institute of Rheumatology, Tokyo Women's Medical University, 10-22 Kawada-cho, Shinjuku-ku, Tokyo 162-0054, Japan
}

associated with SLE, including reporting standards and recommendations for laboratory and imaging tests [2]. Although this standardized nomenclature has helped to clarify a complicated situation, its usefulness as a clinical diagnostic criterion remains to be determined.

While neurological or psychological examinations are still the cornerstones for the diagnosis of CNS lupus, neuroimaging, electroencephalography and cerebrospinal fluid tests are also used [1,2,4-8]. Among conventional and more recent neuroimaging tools, conventional magnetic resonance imaging (MRI) still remains the modality of choice because of its availability and accessibility. Newer tools, such as magnetic resonance spectroscopy (MRS), magnetization transfer imaging (MTI), diffusion weighted imaging (DWI), perfusion weighted imaging 
(PWI), single photon emission computed tomography (SPECT), and positron emission tomography (PET) are still investigational (SPECT has probably been most established among these), although they have provided data to greatly improve our understanding of CNS lupus [4-7]. However, even the diagnostic validity and usefulness of conventional MRI is not yet fully established, because most published studies are small in size $(\mathrm{n}<$ 100 , mostly $<50$ ), retrospective, or based on non-standardized case definitions and some include only NPSLE, not SLE patients without CNS syndromes [2,4-7,9]. In order to overcome these problems of the previous studies we prospectively enrolled the largest number of SLE patients with or without active CNS syndrome which was categorized based on the ACR standardized case definitions and evaluated the accuracy and usefulness of conventional MRI in the diagnosis of CNS syndrome. We also assessed the suitability of conventional MRI as a surrogate marker for CNS lupus.

\section{Methods}

\section{Patient population}

This was a prospective study of 191 SLE patients with or without NP syndromes who were admitted to the Aoyama Hospital of The Tokyo Women's Medical University from August 1994 through October 2003. All these patients had 4 or more revised ACR (formerly, the American Rheumatism Association) criteria for SLE [10]. At our institution, those patients suspected of or newly diagnosed as having SLE were typically admitted for systemic evaluation regardless of the severity of the disease, and were eligible for inclusion in the study. Previously diagnosed SLE patients whose disease (NP syndrome or other manifestations of SLE) flared were also enrolled in the study. A total of 269 patients with SLE gave informed consent for inclusion in this study, including their MRI examinations during the above period. Among these 269 patients, those who had non-SLErelated NP manifestations arising from infection, uremia, electrolyte imbalance, hypoxia, brain tumor, trauma, primary mental disease or drug use $(n=45)$ or past histories of NP involvement $(\mathrm{n}=33)$ were excluded prior to MRI scans. The patients were excluded because we wanted to compare recently (i.e. within a month) diagnosed active CNS lupus patients to non-NPSLE patients and because the unrelated conditions could affect current symptoms or laboratory and MRI findings. At the time of admission to the hospital, each patient completed a standardized medical history, including medication use, and had physical examinations that included neurologic and rheumatologic examinations. Psychiatric examinations were employed when indicated. Serology profiling for each patient was performed using standard immunoassays. The activity of SLE was measured using the SLE Activity Index (SLEDAI) [11]. Treatment with corticosteroids or immunosuppressive drugs was instituted as indicated following completion of these evaluations. One non-NPSLE patient at admission, who later developed NPSLE, was reevaluated at the onset of the NP syndrome (a mood disorder in this patient) and was reclassified into the CNS group. In this patient, data from the reevaluation, including the results of MRI, were used. Subjects were classified into the CNS group or the non-CNS group according to the presence or absence of active CNS syndromes. The CNS group was then further classified into the neurologic disorders group consisting of patients with neurologic disorders with or without other NP syndromes, or the psychiatric disorders group comprising patients with psychiatric disorders with or without other NP syndromes $[2,4,8,12]$. Thirteen patients were classified into both neurologic and psychiatric disorders groups. Detailed diagnostic criteria for these groups are described below. The study was approved by the Ethical Committee of our institution and the Helsinki declaration was followed throughout the study.

\section{Diagnosis of CNS lupus}

Although ACR nomenclature and case definitions include 12 CNS syndromes and 7 peripheral nervous system syndromes $[2,4,8,12]$, we included only the 12 CNS syndromes in the present study because of the substantial differences in anatomy, function, and clinical characteristics between the central and peripheral nervous systems. Slight or mild cognitive dysfunction without significant clinical impairment, as revealed by the detailed neuropsychological tests described below in "Evaluation of psychological impairment," was excluded from the CNS syndromes in our study. Using published normative data, patients in this category $(n=3)$ exhibited less than the median of normal controls by 2 standard deviation (SD) in 2 or more of areas of cognitive function without significant clinical impairment [13]. Tension headache, or episodic tension type headache, was also excluded from our study.

The final clinical diagnosis and classification of the various NP syndromes for inclusion in the study were made by an experienced rheumatologist (M. Hara) and psychiatrist (K. N.), according to the standardized ACR nomenclature and case definitions for neuropsychiatric lupus syndromes [2]. These decisions were based on the medical history and neuropsychological examinations by rheumatologists, an experienced neurologist (S. U.) and a psychiatrist (K. N.) and supported by conventional laboratory tests and appropriate complementary tests, including MRI, electroencephalography, and cerebral spinal fluid tests, and the clinical course. Cases before the ACR nomenclature and case definitions for NPSLE were published in 1999 were originally diagnosed and 
classified according to another criteria and later re-diagnosed retrospectively according to the ACR criteria.

Clinical improvement of CNS lupus was defined as either sustained complete recovery or recovery with minor residual deficits that no longer required hospitalization. Stabilization was defined as the status in which no new clinical (i.e., neurologic or psychiatric) abnormalities occurred, although the previous abnormalities remained. Deterioration was defined as the status in which previous neuropsychiatric symptoms were exacerbated or new ones developed during follow-up $[14,15]$.

\section{Evaluation of psychological impairment}

Cognitive function was evaluated using the Mini Mental Status Examination [16] and the ACR-suggested test battery [2], including the WAIS-R/Digit Span (Forward) [17], Trail Making Test (Part B) [18], WAIS-R/Digit Span (Backward) [17], Wisconsin Card Sorting Test [18], Rey Auditory-Verbal Learning Test [17], WAIS-R/ Block Design [17], Animal Naming Test [18], WAIS-R/ Similarities [17], Trail Making Test (Part A) [18], and WAIS-R/Digit Symbol Substitution Test [17]. Mood and behavioral dysfunction was assessed by clinical observation, patient history and standardized instruments, such as the Profile of Mood States [19]. However, these formal neuropsychological tests were not performed routinely in all patients and the diagnosis of cognitive, mood, or behavioral dysfunction was on the basis of clinical assessment using the ACR definitions [2], rather than formal neuropsychological testing, especially in cases where disturbance of consciousness, such as acute confusional state, created difficulty in taking the tests. The impact of disturbance on daily life and prior occupational and social functioning was determined from information provided by the patient or other informants.

\section{Magnetic resonance imaging (MRI)}

MRI was performed using a $1.5 \mathrm{~T}$ MR scanner (Toshiba Medical Systems, Otawara, Tochigi, Japan) within a week from admission and within a month from onset of NP symptoms in the CNS group. Scans were aligned parallel to the axial plane through the anterior to posterior commissure and covered the entire brain in all sequences. T1-weighted, T2-weighted, and fluid-attenuated inversion recovery (FLAIR) images were acquired from all patients who had brain MRIs. The software, but not the hardware of the MR scanner was updated once in 2000, which improved its resolution but did not practically influence the sensitivity of this study. The MRIs were interpreted at the time of scanning by an experienced radiologist (T. Y.) who was not blinded to all the clinical and sequential information. MRI tests were defined as 'positive' when any abnormal intensity lesion was found. The abnormal MRI signals were classified into large abnormal MRI signals $(\varnothing \geq 10 \mathrm{~mm}$ ) or small abnormal MRI signals $(\varnothing<10 \mathrm{~mm}$ ) [15]. Mild brain atrophy, characterized by loss of brain volume, was not included among MRI abnormalities in the present study because it is not a well-established abnormality [6], even though it is the most frequent abnormal findings in SLE [9]. Moderate to severe brain atrophy was not seen at the time of enrolment in the study population. CNS lupus patients who had had abnormalities in initial MRIs were reevaluated by MRI at approximately 1 year or when clinical amelioration or deterioration was determined. MRI improvement was defined as a more than $50 \%$ decrease in the number or size of abnormal MRI signals. MRI stabilization was defined as occurring when no new abnormalities were detected, but previously detected abnormalities were unchanged or only slightly changed. Deterioration was defined as when the previously detected abnormalities became exacerbated or new ones developed during follow-up [15].

\section{Statistical analyses}

Diagnostic tests evaluated in the present study were correlated with the final clinical diagnosis for each. Twogroup comparisons were analyzed using the MannWhitney $\mathrm{U}$ test for continuous variables and Fisher's exact test for categorical variables. Values of $p<0.05$ were considered statistically significant. Accuracy, positive predictive value (PPV), and negative predictive value (NPV) of MRI and the relative risk of CNS lupus to MRI were also calculated. All statistical analyses were performed using SPSS statistical software (version 14.0J; SPSS Inc., Tokyo, Japan).

\section{Results}

\section{Clinical characteristics of the patients}

Of the 191 patients with SLE enrolled in the present study, 176 were women and 15 were men. The median age of the patients was 32 years (range; 11 to 68 years). The median disease duration since the diagnosis of SLE was 1 year (range; 0 to 22 years). The patients were all Japanese, except for 1 woman who was Chinese. Current CNS syndromes were observed in 57 patients (CNS group), while the remaining 134 patients did not have either current CNS syndromes or a history of CNS syndromes (non-CNS group). Detailed clinical characteristics of the study patients are summarized in Table 1. Arthritis was significantly more frequent in the nonCNS group ( $p=0.036)$ and levels of serum anti-dsDNA antibody were also significantly higher in the non-CNS group ( $p=0.035)$. Although the SLEDAI score differed significantly between the 2 groups $(p<0.0001)$, the "SLEDAI score without CNS syndrome," determined by evaluating and summing the clinical variables of the SLEDAI score other than CNS syndrome for each patient, was not (Table 1 ).

Table 2 shows the distribution of types of CNS syndrome in the study group. Neurologic disorders alone 
Table 1 Characteristics of the 191 patients with SLE

\begin{tabular}{|c|c|c|c|}
\hline & CNS Group & Non-CNS Group & $p \dagger$ \\
\hline Characteristics & $\mathrm{n}=57$ & $\mathrm{n}=134$ & \\
\hline Female/male & $53 / 4$ & $123 / 11$ & 1.000 \\
\hline Age at evaluation (yrs) & $28:[23,43]$ & $34:[25,45]$ & 0.160 \\
\hline SLE duration at evaluation (yrs) & $1:[0,5]$ & $1:[0,4]$ & 0.322 \\
\hline \multicolumn{4}{|l|}{ Clinical features } \\
\hline Malar rash/discoid rash & $25(44 \%)$ & $42(31 \%)$ & 0.101 \\
\hline Oral or nasal ulcers & $1(2 \%)$ & $13(10 \%)$ & 0.068 \\
\hline Arthritis & $16(28 \%)$ & $60(45 \%)$ & 0.036 \\
\hline Serositis & $7(12 \%)$ & $18(13 \%)$ & 1.000 \\
\hline Renal disorder & 19 (33\%) & $37(28 \%)$ & 0.488 \\
\hline Vasculitis & $1(2 \%)$ & $7(5 \%)$ & 0.439 \\
\hline Antinuclear antibody & $52(95 \%)$ & $131(98 \%)$ & 0.150 \\
\hline Antiphospholipid antibodiesł & $13(23 \%)$ & $39(29 \%)$ & 0.478 \\
\hline Lymphocytopenia $\left(<1500 / \mathrm{mm}^{3}\right.$ ) & $44(80 \%)$ & $91(69 \%)$ & 0.154 \\
\hline SLEDAI & $15:[10,22]$ & $9:[5,11]$ & $<0.0001$ \\
\hline SLEDAI without CNS score & $11:[7,16]$ & $9:[5,11]$ & 0.606 \\
\hline Anti-dsDNA antibody (RIA; IU/ml) & $8:[3,37]$ & $20:[5,98]$ & 0.035 \\
\hline $\mathrm{CH} 50(\mathrm{U} / \mathrm{ml})$ & $33.2:[21.2,39.2]$ & $29.8:[20.4,38.3]$ & 0.496 \\
\hline
\end{tabular}

*Except where indicated otherwise, values of continuous data are the median : [25\% percentile, $75 \%$ percentile]. $\dagger P$ values were determined by Fisher's exact test or Mann-Whitney $U$ test.

¥Antiphospholipid antibodies include lupus anticoagulant, anti-cardiolipin antibodies, and anti- $\beta 2 \mathrm{GPI}$ antibodies.

CNS: central nervous system; SLEDAI: systemic lupus erythematosus disease activity index; RIA: radioimmunoassay.

were diagnosed in 34 subjects, psychiatric disorders alone in 36 , and both in 13 . CNS syndromes in patients with both disorders were seizure disorders $(n=7)$, demyelinating syndrome $(\mathrm{n}=4)$, aseptic meningitis $(\mathrm{n}=$ $2)$, acute confusional state $(n=11)$, anxiety disorder $(n$ $=1)$ and psychosis $(n=1)$. The most frequent manifestation of neurologic disorders was seizures and seizure disorders $(\mathrm{n}=18)$, while that of psychiatric disorders was acute confusional state $(n=24)$.

\section{Initial MRI}

All patients enrolled in the present study underwent brain MRI. We assessed the diagnostic value of brain MRI for CNS lupus using this data. Abnormal MRI signals were more frequently observed in the CNS group $(\mathrm{n}=25,44 \%)$ than in the non-CNS group $(\mathrm{n}=32,25 \%)$ with significant statistical differences [relative risk (RR), 1.7; 95\% confidence interval (CI), 1.1-2.7; $p=0.016$ ] (Table 3). This resulted in a diagnostic accuracy of $65 \%$, a PPV of $42 \%$ and a NPV of $76 \%$ for MRI use in diagnosis of CNS lupus. Significant differences were also found when the CNS group with neurologic disorders $(n=17$; RR, 2.3; CI, 1.3-4.1; $p=0.007$ ) and the CNS group with psychiatric disorders $(\mathrm{n}=16$; $\mathrm{RR}, 1.9 ; \mathrm{CI}, 1.1-3.4 ; p=$ 0.038 ) were separately compared with the non-CNS group.

Because the predictive values, in particular the PPV, of MRI were not considered adequate for the practical diagnosis of CNS lupus, we then analyzed the comparative sizes of abnormal MRI signals. Abnormal MRI signals on T2-weighted or FLAIR images of the brains of SLE patients were usually small nonspecific fixed foci of increased signal in deep white matter (Figure 1A). Midto large-sized high intensity lesions in brain T2weighted or FLAIR images were occasionally observed, but only in the CNS group (Figure 1B). When abnormal signals in MRI were classified by size, large signals $(\varnothing \geq$ $10 \mathrm{~mm})$ were seen only in the CNS group $(\mathrm{n}=7 ; 12 \%$; RR, 3.7; CI, 2.9-4.7; $p=0.0002$ ) (Table 4). Smaller signals $(\varnothing<10 \mathrm{~mm}$; mostly $\varnothing<5 \mathrm{~mm})$ were seen in both the CNS and non-CNS groups with no significant difference $(\mathrm{n}=18 ; 32 \%$ and $34 ; 25 \%$, respectively; $\mathrm{RR}, 1.2 ; \mathrm{CI}$, $0.8-2.0 ; p=0.380$ ) (Table 4). Calculations showed 74\% accuracy, a PPV of $100 \%$ and an NPV of $73 \%$ using large abnormal MRI signals for the diagnosis of CNS lupus. Clinical syndromes of the patients showing large abnormal MRI signals included seizure disorders $(\mathrm{n}=$ $2)$, cerebrovascular disease $(n=1)$, demyelinating syndrome $(\mathrm{n}=3)$, and acute confusional state $(\mathrm{n}=5)$ (Table 5).

\section{Chronological changes in abnormal MRI signals}

The association between chronological changes of abnormal MRI signals and the clinical outcome in the CNS group was examined. As shown in Tables 5 and 6 , large signals always decreased in size or resolved 
Table 2 CNS syndromes of SLE patients $(n=191)$

\begin{tabular}{llcc}
\hline Manifestaions of CNS lupus* & $\mathbf{n}$ & $\%$ & MRI positivet \\
\hline Total & 57 & 30 & $25(44 \%)$ \\
Neurologic disorders & & 18 & $17(50 \%)$ \\
Aseptic meningitis & 34 & 3 & $1(20 \%)$ \\
Cerebrovascular disease & 5 & 1 & $1(100 \%)$ \\
Demyelinating syndrome & 1 & 3 & $4(80 \%)$ \\
Headacheł & 5 & 3 & $4(80 \%)$ \\
Movement disorder (chorea) & 5 & 0 & $1(50 \%)$ \\
Myelopathy & 0 & 1 & $8(44 \%)$ \\
Seizures and seizure disorders & 2 & 9 & $16(44 \%)$ \\
Psychiatric disorders & 18 & 19 & $14(58 \%)$ \\
Acute confusional state & & 13 & $0(0 \%)$ \\
Anxiety disorder & 36 & 1 & $2(22 \%)$ \\
Cognitive dysfunction§ & 24 & 0 & $0(0 \%)$ \\
Mood disorders & 1 & 5 & 1 \\
Psychosis & 0 & 9 & \\
\hline
\end{tabular}

* Based on American College of Rheumatology case definitions for neuropsychiatric ayndromes in SLE

†An MRI test was defined to be 'positive' when any abnormal intensity lesion was found.

\#Excluding tension headache (episodic tension-type headache).

$\S$ Excluding slight or mild cognitive dysfunction without significant clinical impairment as revealed by detailed neuropsychological tests.

SLE: systemic lupus erythematosus; CNS: central nervous system

completely in the 4 subjects where CNS syndromes were ameliorated by treatment (a representative case is shown in Figure $1 \mathrm{~B}$ and $1 \mathrm{C}$ ), but were unchanged in the 3 subjects where CNS syndromes did not improve; this difference was statistically significant $(p=0.029)$. Chronological changes in small signals did not correspond with clinical outcome.

\section{Discussion}

First, from the largest prospective cohort of SLE patients with or without active CNS syndrome, we found that abnormal signals from conventional MRI alone did not have sufficient predictive value to be practical for diagnosis of active CNS lupus, although they were associated with active CNS lupus. Second, after assortment by size, large signals $(\varnothing \geq 10 \mathrm{~mm})$ were found to occur only in the CNS group while small signals $(\varnothing<10 \mathrm{~mm})$ occurred in both the CNS and non-CNS groups with no significant difference in frequency. Finally, we did find that large signals were always reduced or resolved completely when CNS syndromes were ameliorated by treatment but did not change when CNS syndromes did not improve. In contrast, chronological changes in small signals were not related to clinical outcome. We believe this is the first report analyzing predictive values of MRI for diagnosis of active CNS lupus using a prospective study of a large cohort of patients with and without NP syndromes.

This study demonstrated that active CNS lupus was not associated with elevated "SLEDAI scores without CNS syndrome," elevated anti-DNA antibody levels, or decreased serum $\mathrm{CH} 50$ levels. The latter two are widely recognized and clinically used as reliable laboratory tests for estimating disease activity in patients with SLE $[20,21]$. Our data support the common view that CNS syndrome in SLE may occur independently from and in

Table 3 MRI as a diagnostic tool for CNS lupus in 191 patients with SLE

\begin{tabular}{|c|c|c|c|c|c|c|}
\hline & MRI positive & MRI negative & $\begin{array}{l}\text { Relative risk } \\
\quad(95 \% \mathrm{Cl})\end{array}$ & $p$ & PPV & NPV \\
\hline CNS Group & $25(44 \%)$ & $32(56 \%)$ & $1.7(1.1-2.7)$ & 0.016 & 0.424 & 0.758 \\
\hline Neurologic disorders & $17(50 \%)$ & $17(50 \%)$ & $2.3(1.3-4.1)$ & 0.007 & 0.333 & 0.855 \\
\hline Psychiatric disorders & $16(44 \%)$ & $20(56 \%)$ & $1.9(1.1-3.4)$ & 0.038 & 0.320 & 0.833 \\
\hline Non-CNS Group & $34(25 \%)$ & $100(75 \%)$ & - & - & - & - \\
\hline
\end{tabular}

*An MRI test was defined to be 'positive' when any abnormal intensity lesion was found.

$+P$ values were determined by Fisher's exact test and CNS group or its subgroup is compared with non-CNS group.

$\mathrm{Cl}$ : Confidence Interval; PPV: positive predictive value; NPV: negative predictive value 

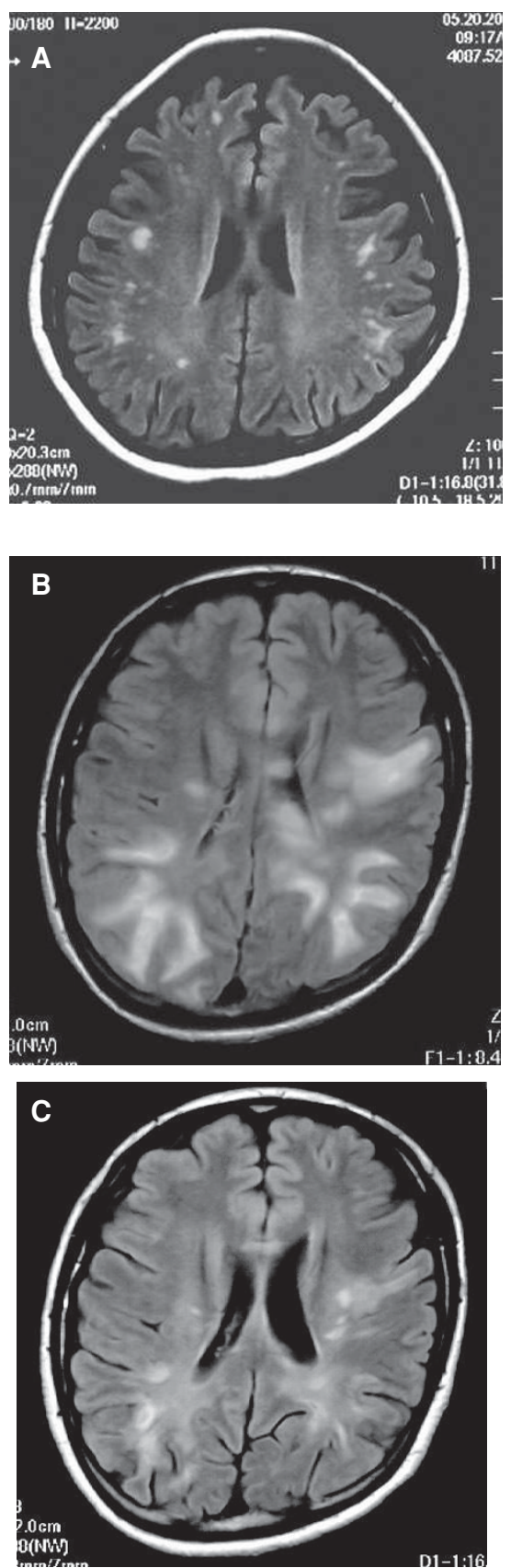

Figure 1 Abnormal magnetic resonance imaging signals in patients with systemic lupus erythematosus (SLE). (A) Typical white-matter lesions in central nervous system (CNS) lupus in a fluid-attenuated inversion-recovery (FLAIR) image of a 21-year-old woman having a headache caused by benign intracranial hypertension. Many foci of small-sized abnormal signals are visible in the white-matter of the frontal and parietal lobes. This type of abnormality was also observed in some of the SLE patients who had no history of CNS syndrome. (B) FLAIR image of a 23-year-old woman with demyelinating syndrome and acute confusional state, showing multiple large hyperintensities involving both grey and white matter (Case 3 in Table 5). (C) Remarkable resolution of the clinical signs and imaging picture 3 months later, following high dose glucocorticoid with intravenous cyclophosphamide therapy. the absence of serological activity or other organ involvement [22].

Although we observed abnormal signal intensities in the brain MRIs of the CNS group more frequently than in those of the non-CNS group, the PPV (42\%) and the NPV (76\%) of MRI for detecting active CNS lupus indicate that conventional MRI alone is insufficiently specific to serve as a diagnostic tool for active CNS lupus. Active CNS lupus was diagnosed in less than half of the patients with abnormal signal intensities in brain MRI, even though normal MRI findings tended to be associated with non-CNS lupus. We also categorized patients by CNS manifestations indicating neurologic or psychiatric disorders but did not find any better results. In fact, MRI has been shown to be neither very sensitive nor specific for the diagnosis of NPSLE, estimates of sensitivity and specificity being in the 30 and $40 \%$, respectively [12]. We would like to point out that these figures had been only estimates without solid evidence until this study. Thus, the diagnosis of CNS syndrome in SLE remains a difficult task requiring careful clinical and laboratory assessment together with such evaluation techniques as neuroimaging, electroencephalography and cerebrospinal fluid tests $[7,12]$.

While exploring the usefulness of conventional MRI, we found that a large MRI signal is useful as a diagnostic and surrogate marker for CNS lupus. Large abnormal MRI signals $(\varnothing \geq 10 \mathrm{~mm})$ were seen only in the CNS group whereas small abnormal MRI signals ( $<10 \mathrm{~mm}$ ) were seen in both groups with no significant difference. In addition, chronological changes in large signals always corresponded with clinical outcome while those of small signals did not. This kind of reversible large abnormal MRI signals has been reported $[4,12,15,23,24]$. These large abnormal MRI signals may include new infarcts, discrete gray matter lesions, diffuse gray matter hyperintensities, and cerebral edema. These lesions, except for infarcts, are often transitory and resolve completely with time and treatment. The large abnormal MRI signals in the present study included several types of features, such as infarcts, diffuse gray matter hyperintensities, and widespread grey and white matter T2 high signal changes resembling those in acute disseminated encephalomyelitis, accompanied by various CNS syndromes. Although these large abnormal MRI signals were attributed to different pathomechanisms, they exhibited equal usefulness as a diagnostic and surrogate marker. Therefore, our simple criterion (i.e., MRI signals with $\emptyset \geq 10 \mathrm{~mm}$ ) is applicable to daily clinical practice without specialized knowledge of neuroimaging and regardless of the type of CNS syndromes encountered. We believe this test will prove very beneficial for clinical assessment of the condition. 
Table 4 Large MRI signal as a diagnostic tool for CNS lupus in 191 patients with SLE

\begin{tabular}{|c|c|c|c|c|c|c|}
\hline & Large signal & Negative or small signal & $\begin{array}{c}\text { Relative risk } \\
(95 \% \mathrm{Cl})\end{array}$ & $p$ & PPV & NPV \\
\hline CNS group & $7(12 \%)$ & $50(88 \%)$ & $3.7(2.9-4.7)$ & 0.0002 & 1.000 & 0.728 \\
\hline Neurologic Disorders & $5(15 \%)$ & $29(85 \%)$ & $5.6(4.0-7.8)$ & 0.0003 & 1.000 & 0.822 \\
\hline Psychiatric Disorders & $5(14 \%)$ & $31(86 \%)$ & $5.3(3.9-7.3)$ & 0.0003 & 1.000 & 0.812 \\
\hline Non-CNS group & $0(0 \%)$ & $134(100 \%)$ & - & - & - & - \\
\hline
\end{tabular}

*Abnormal MRI signals were classified into large $(\varnothing \geq 10 \mathrm{~mm}$ ) or small abnormal MRI signals $(\varnothing<10 \mathrm{~mm})$.

$+P$ values were determined by Fisher's exact test and CNS group or its subgroup is compared with non-CNS group.

$\mathrm{Cl}$ : Confidence Interval; PPV: positive predictive value; NPV: negative predictive value

In agreement with our present findings, small punctate focal lesions in the white matter have been reported to be the most common brain MRI finding, tending to be more frequently observed in the CNS than in the non-CNS SLE patients in most published reports $[6,7,12,25,26]$. These lesions appear anywhere in the brain, including the brain stem, and are accompanied by a variety of clinical presentation. These small punctuate hyperintense T2-weighted focal lesions are frequently nonspecific and interpreted as consistent with focal ischemia, demyelination, vasculitis, microinfarcts, gliosis, or other conditions. These small abnormal MRI signals frequently correlate poorly with clinical manifestations, and occur in patients without marked CNS signs and symptoms. In addition, conventional MRI is not capable of differentiating between older chronic and recent acute lesions of this type.

Neuroimaging must be evaluated in the context of the brain pathology of CNS lupus [4]. At this time, pathologic studies have basically been limited to post mortem studies of end stage SLE patients, which has seriously limited understanding of the sequence of events in the pathogenesis and progression of the disease [5]. Moreover, there have been few paired imaging-autopsy studies [4]. Despite the dramatic neurologic manifestations, minimal histopathologic findings in CNS lupus are typical, with apparently normal findings or nonspecific changes in the brain predominating. This general lack of specific histopathology has complicated interpretation of results found with nearly any imaging technique. For example, the autopsy of one of the patients in the present study who died from alveolar hemorrhage 5 years after showing acute confusional state and several foci of small-sized abnormal MRI signals in the white-matter showed no remarkable findings in the brain.

One of the limitations of the present study is that its subject number was insufficient for definitive conclusions about significance of conventional MRI for diagnosis of CNS lupus, although it is appreciably larger than previous studies dealing with NPSLE and MRI $[15,24,27]$. Another potential weakness arises from the fact that the MRI scans were not evaluated by multiple

Table 5 Characteristics of the CNS lupus patients with large abnormal MRI signals

\begin{tabular}{|c|c|c|c|c|c|c|c|c|}
\hline Case & $\begin{array}{l}\text { Duration of } \\
\text { SLE (yrs) }\end{array}$ & SLEDAI & $\begin{array}{c}\text { SLEDAI } \\
\text { without } \\
\text { CNS }\end{array}$ & $\mathrm{aPL} *$ & SLE-related CNS syndrome & Treatment & $\begin{array}{l}\text { Clinical outcome } \\
\text { of CNS lupus }\end{array}$ & $\begin{array}{c}\text { MRI } \\
\text { change }\end{array}$ \\
\hline 1 & 0 & 31 & 15 & Positive & Seizure disorder, Headache & Steroids & Improve & Improve \\
\hline 2 & 0 & 11 & 3 & Negative & Acute confusional state & Steroids + CPA & Improve & Improve \\
\hline 3 & 1 & 24 & 8 & Negative & $\begin{array}{l}\text { Demyelinating syndrome, Acute } \\
\text { confusional state }\end{array}$ & Steroids + CPA & Improve & Improve \\
\hline 4 & 9 & 29 & 5 & Positive & $\begin{array}{l}\text { Demyelinating syndrome, Seizure } \\
\text { disorder, Acute confusional state }\end{array}$ & Steroids + CPA & Improve & Improve \\
\hline 5 & 4 & 16 & 0 & Negative & $\begin{array}{l}\text { Demyelinating syndrome, Acute } \\
\text { confusional state }\end{array}$ & Steroids + CPA & No change & No change \\
\hline 6 & 8 & 10 & 2 & Negative & Cerebrovascular disease & $\begin{array}{l}\text { Steroids + CPA + } \\
\text { anticoagulant }\end{array}$ & No change & No change \\
\hline 7 & 5 & 22 & 6 & Negative & Acute confusional state & Steroids + CPA & No change & Deteriorate \\
\hline
\end{tabular}

CNS: central nervous system; MRI: magnetic resonance imaging; SLE: systemic lupus erythematosus; SLEDAl: systemic lupus erythematosus disease activity index; aPL: antiphospholipid antibody; CPA: cyclophosphamide.

*aPL includes lupus anticoagulant, anti-cardiolipin antibodies, and anti- $\beta 2 \mathrm{GPI}$ antibodies.

tThe case numbers given are not identifying numbers. 
Table 6 Large or small MRI signal as a surrogate marker for CNS lupus in 25 SLE patients

\begin{tabular}{|c|c|c|c|c|}
\hline \multirow[b]{2}{*}{ MRI signal size } & \multirow[b]{2}{*}{ MRI change } & \multicolumn{2}{|c|}{ Clinical outcome of CNS syndrome } & \multirow[b]{2}{*}{$p$} \\
\hline & & Improve & Stabilize or deteriorate & \\
\hline \multirow[t]{2}{*}{ Large } & Improve & 4 & 0 & 0.029 \\
\hline & Stabilize or deteriorate & 0 & 3 & \\
\hline \multirow[t]{2}{*}{ Small } & Improve & 2 & 0 & 1.000 \\
\hline & Stabilize or deteriorate & 13 & 3 & \\
\hline
\end{tabular}

*P values were determined by Fisher's exact test.

†Large MRI signal size $=\varnothing \geq 10 \mathrm{~mm}$; Small MRI signal size $=\varnothing<10 \mathrm{~mm}$, mostly $\varnothing<5 \mathrm{~mm}$

CNS: central nervous system; MRI: magnetic resonance imaging; SLE: systemic lupus erythematosus; PPV: positive predictive value; NPV:negative predictive value

radiologists blinded to the clinical condition. Fortunately, it has been shown that evaluation of these scans is generally consistent among observers, so cranial MRI may be considered to be a valid technique to assess disease progression or reversal [15]. Because we did not include SLE patients who had CNS syndromes caused by non-SLE pathologies, the value of MRI for diagnosis of these conditions was not determined by this study. Thus, it is not clear from this study whether CNS syndromes caused by non-SLE pathologies can be differentiated by MRI, including the large MRI abnormal signals.

\section{Conclusions}

MRI alone does not have sufficient predictive value for the diagnosis of active CNS lupus, although abnormal MRI signals showed a significant association with CNS syndrome. Importantly, a large MRI signal is a reliable and practical diagnostic and surrogate marker for CNS syndrome in SLE. The relatively small number of subjects having large MRI signals in the study indicates the need for further studies in larger numbers of patients to validate the findings.

\section{Acknowledgements}

We thank Dr. Masashi Uesato (Tokyo Women's Medical University) for assisting in construction of the database of SLE patients and Professor Shinichiro Uchiyama (Department of Neurology, Neurological Institute, Tokyo Women's Medical University) for assisting with the neurological examinations and diagnoses.

\section{Author details \\ ${ }^{1}$ Institute of Rheumatology, Tokyo Women's Medical University, 10-22 Kawada-cho, Shinjuku-ku, Tokyo 162-0054, Japan. ${ }^{2}$ Department of Pharmacovigilance, Graduate School, Tokyo Medical and Dental University, 1- 5-45 Yushima, Bunkyo-ku, Tokyo 113-8519, Japan. ${ }^{3}$ Department of Psychiatry, Tokyo Women's Medical University School of Medicine, 8-1 Kawada-cho, Shinjuku-ku, Tokyo 162-8666, Japan. ${ }^{4}$ Department of Radiology, Tokyo Women's Medical University School of Medicine, 8-1 Kawada-cho, Shinjuku- ku, Tokyo 162-8666, Japan.}

\section{Authors' contributions}

Y Katsumata conceived of the study and drafted the manuscript. M Harigai participated in data analysis and interpretation, and helped to draft the manuscript. Y Kawaguchi, CF, MS and TK participated in acquisition of data and patient recruitment. KN was responsible for psychological assessment.
TY was responsible for MRI analyses. HY and M Hara participated in the design and coordination of the study. All authors read and approved the final manuscript.

\section{Competing interests}

The authors declare that they have no competing interests.

Received: 11 December 2009

Accepted: 23 January 2010 Published: 23 January 2010

\section{References}

1. Hanly JG, Harrison MJ: Management of neuropsychiatric lupus. Best Pract Res Clin Rheumatol 2005, 19(5):799-821.

2. The American College of Rheumatology nomenclature and case definitions for neuropsychiatric lupus syndromes. Arthritis Rheum 1999, 42(4):599-608.

3. Nishimura K, Harigai M, Omori M, Sato E, Hara M: Blood-brain barrier damage as a risk factor for corticosteroid-induced psychiatric disorders in systemic lupus erythematosus. Psychoneuroendocrinology 2008, 33(3):395-403.

4. Sibbitt WL Jr, Sibbitt RR, Brooks WM: Neuroimaging in neuropsychiatric systemic lupus erythematosus. Arthritis Rheum 1999, 42(10):2026-2038.

5. Huizinga TW, Steens SC, van Buchem MA: Imaging modalities in central nervous system systemic lupus erythematosus. Curr Opin Rheumatol 2001, 13(5):383-388.

6. Appenzeller S, Pike GB, Clarke AE: Magnetic resonance imaging in the evaluation of central nervous system manifestations in systemic lupus erythematosus. Clin Rev Allergy Immunol 2008, 34(3):361-366.

7. Castellino G, Govoni M, Giacuzzo S, Trotta F: Optimizing clinical monitoring of central nervous system involvement in SLE. Autoimmun Rev 2008, 7(4):297-304.

8. Katsumata Y, Harigai M, Kawaguchi Y, Fukasawa C, Soejima M, Takagi K, Tanaka M, Ichida H, Tochimoto A, Kanno T, et al: Diagnostic reliability of cerebral spinal fluid tests for acute confusional state (delirium) in patients with systemic lupus erythematosus: interleukin 6 (IL-6), IL-8, interferon-alpha, IgG index, and Q-albumin. J Rheumatol 2007, 34(10):2010-2017.

9. Petri M, Naqibuddin M, Carson KA, Wallace DJ, Weisman MH, Holliday SL, Sampedro M, Narayana S, Fox PT, Franklin C, et al: Brain magnetic resonance imaging in newly diagnosed systemic lupus erythematosus. $J$ Rheumatol 2008, 35(12):2348-2354.

10. Tan EM, Cohen AS, Fries JF, Masi AT, McShane DJ, Rothfield NF, Schaller JG, Talal N, Winchester RJ: The 1982 revised criteria for the classification of systemic lupus erythematosus. Arthritis Rheum 1982, 25(11):1271-1277.

11. Bombardier C, Gladman DD, Urowitz MB, Caron D, Chang CH: Derivation of the SLEDAI. A disease activity index for lupus patients. The Committee on Prognosis Studies in SLE. Arthritis Rheum 1992, 35(6):630-640.

12. Jennings JE, Sundgren PC, Attwood J, McCune J, Maly P: Value of MRI of the brain in patients with systemic lupus erythematosus and neurologic disturbance. Neuroradiology 2004, 46(1):15-21.

13. Kozora E, Thompson LL, West SG, Kotzin BL: Analysis of cognitive and psychological deficits in systemic lupus erythematosus patients without overt central nervous system disease. Arthritis Rheum 1996, 39(12):2035-2045. 
14. Neuwelt CM, Lacks S, Kaye BR, Ellman JB, Borenstein DG: Role of intravenous cyclophosphamide in the treatment of severe neuropsychiatric systemic lupus erythematosus. Am J Med 1995 98(1):32-41.

15. Karassa FB, loannidis JP, Boki KA, Touloumi G, Argyropoulou MI, Strigaris KA, Moutsopoulos HM: Predictors of clinical outcome and radiologic progression in patients with neuropsychiatric manifestations of systemic lupus erythematosus. Am J Med 2000, 109(8):628-634.

16. American Psychiatric Association: Diagnostic and Statistical Manual of Mental Disorders. American Psychiatric Association, 41994.

17. Wechsler D: Wechsler Adult Intelligence Scale - Revised. New York: The Psychological Corporation 1981

18. Lezak MD: Neuropsychological assessment. New York: Oxford University Press, 31997.

19. McNair DM, Lorr M, Droppleman LF: Manual for the Profile of Mood States. San Diego: Educational and Industrial Testing Service 1992.

20. Merrill JT, Buyon JP: The role of biomarkers in the assessment of lupus. Best Pract Res Clin Rheumatol 2005, 19(5):709-726.

21. Mills JA: Systemic lupus erythematosus. N Engl J Med 1994, 330(26):1871-1879.

22. Winfield JB, Brunner CM, Koffler D: Serologic studies in patients with systemic lupus erythematosus and central nervous system dysfunction. Arthritis Rheum 1978, 21(3):289-294.

23. Graham JW, Jan W: MRI and the brain in systemic lupus erythematosus. Lupus 2003, 12(12):891-896.

24. Sibbitt WL Jr, Sibbitt RR, Griffey RH, Eckel C, Bankhurst AD: Magnetic resonance and computed tomographic imaging in the evaluation of acute neuropsychiatric disease in systemic lupus erythematosus. Ann Rheum Dis 1989, 48(12):1014-1022.

25. Sabbadini MG, Manfredi AA, Bozzolo E, Ferrario L, Rugarli C, Scorza R, Origgi L, Vanoli M, Gambini O, Vanzulli L, et al: Central nervous system involvement in systemic lupus erythematosus patients without overt neuropsychiatric manifestations. Lupus 1999, 8(1):11-19.

26. Sanna G, Piga M, Terryberry JW, Peltz MT, Giagheddu S, Satta L, Ahmed A Cauli A, Montaldo C, Passiu G, et al: Central nervous system involvement in systemic lupus erythematosus: cerebral imaging and serological profile in patients with and without overt neuropsychiatric manifestations. Lupus 2000, 9(8):573-583.

27. Steens SC, Admiraal-Behloul F, Bosma GP, Steup-Beekman GM, Olofsen H, Le Cessie S, Huizinga TW, Van Buchem MA: Selective gray matter damage in neuropsychiatric lupus. Arthritis Rheum 2004, 50(9):2877-2881.

\section{Pre-publication history}

The pre-publication history for this paper can be accessed here:http://www. biomedcentral.com/1471-2474/11/13/prepub

doi:10.1186/1471-2474-11-13

Cite this article as: Katsumata et al.: Diagnostic reliability of magnetic resonance imaging for central nervous system syndromes in systemic lupus erythematosus: a prospective cohort study. BMC Musculoskeletal Disorders 2010 11:13.

\section{Publish with Biomed Central and every scientist can read your work free of charge}

"BioMed Central will be the most significant development for disseminating the results of biomedical research in our lifetime. "

Sir Paul Nurse, Cancer Research UK

Your research papers will be:

- available free of charge to the entire biomedical community

- peer reviewed and published immediately upon acceptance

- cited in PubMed and archived on PubMed Central

- yours - you keep the copyright 